\title{
Optimization of Phototrophic Growth and Lipid Production of a Newly Isolated Microalga, Desmodesmus sp. KAERI-NJ5
}

\author{
Min-Ho Joe ${ }^{1,2}$, Dong-Ho Kim¹ ${ }^{1}$ Dae Seong Choi ${ }^{1}$, and Suk Bai ${ }^{3 *}$ \\ ${ }^{1}$ Department of Biotechnology, Korea Atomic Energy Research Institute, Jeongeup 56212, Republic of Korea \\ ${ }^{2}$ School of Sciences and Biotechnology, ${ }^{3}$ Department of Biological Sciences, College of Natural Sciences, Chonnam National University, \\ Gwangju 61186, Republic of Korea
}

Received: August 6, 2018 / Revised: September 4, 2018 / Accepted: September 5, 2018

In this study, a novel microalgal strain, Desmodesmus sp. KAERI-NJ5, was isolated, identified, and evaluated as a candidate for biodiesel feedstock. In a preliminary study, the effects of four general microalgal growth factors, including temperature, $\mathrm{pH}$, light intensity, and concentration of nitrogen source $\left(\mathrm{KNO}_{3}\right)$, on the microalgal photoautotrophic growth were evaluated. With the exception of light intensity, the growth factors needed to be optimized for the microalgal biomass production. Optimization was done using response surface methodology. The optimal conditions for biomass production were $\mathrm{pH} 6.54,27.66^{\circ} \mathrm{C}$, and $0.52 \mathrm{~g} / \mathrm{KNO}_{3}$. The biomass production at the optimal conditions was $1.55 \mathrm{~g} / \mathrm{l}$, which correlated well with the predicted value of $1.5 \mathrm{~g} / \mathrm{l}$. The total lipid and fatty acid methyl ester contents of the cells grown at the optimal conditions were $49 \%$ and $21.2 \%$ of cell dry weight, respectively. To increase the lipid content of the biomass, microalgae were challenged by nitrogen starvation. Enhancement of total lipid and fatty acid content up to $52.02 \%$ and $49 \%$, respectively, were observed. Lipid analysis of the nitrogen-starved cells revealed that $\mathrm{C} 16$ and $\mathrm{C} 18$ species accounted for $95.9 \%$ of the total fatty acids. Among them, palmitic acid (46.17\%) and oleic acid (39.43\%) dominantly constituted the algal fatty acids. These results suggest Desmodesmus sp. KAERI-NJ5 as a promising feedstock for biodiesel production.

Keywords: Microalgae, Desmodesmus sp. KAERI-NJ5, biomass production, response surface methodology, biodiesel feedstock

\section{Introduction}

Biofuels, along with solar and wind energy, have received tremendous attention due to the increasing demand for fossil fuels and associated environmental problems. Among the various biofuels, biodiesel has been extensively produced from oilseed crops such as soybean, rapeseed, and oil palm [1]. However, biodiesel

\section{*Corresponding author}

Tel: +82-62-530-3412, Fax: +82-62-530-3419

E-mail: sukbai@jnu.ac.kr

() 2018, The Korean Society for Microbiology and Biotechnology production using oilseed crops have critical drawbacks including low yields, high land and water requirements, detrimental effects on food supplies and threats to native biodiversity [2]. Therefore, research on sustainable alternative lipid-rich biomaterials other than traditional oilseed crops has attracted great attention to meet the increasing demand.

Microalgae have been considered as good candidate feedstocks for biodiesel production because of their higher photosynthetic efficiency, higher biomass production and faster growth compared to those of other energy crops. Furthermore, microalgal cultivation systems use 
far less water and land areas than the conventional crops [3]. As a result of these distinct advantages, microalgae are able to produce more oil per unit area of land compared to terrestrial oilseed crops [4]. A key consideration for microalgae-based biodiesel is the selection of appropriate strains. Lipid content, growth rate, ease of cultivation and harvest, and adaptability in local environments should be carefully considered. In addition, the local climate and land geology of different areas should also be considered [5-7]. Although hundreds of microalgae strains have been reported from many screening programs around the world surveying microalgal species in different locations, only very few strains, including Chlorella, Dunaliella, Chlamydomonas, and Nannochloropsis, have been recognized as useful for lipid production $[1,8]$.

South Korea has a temperate climate with four distinct seasons. Winters are usually longer than other seasons, and the temperature deviation between night and day in springs and autumn is usually large. Therefore, exploiting the indigenous microalgae species with desirable characteristics is especially valuable in developing a regional biodiesel industry. Furthermore, most of the fossil fuels consumed in South Korea are imported from abroad because of the lack of natural resources, which indicates significant vulnerability to energy security issues [9]. As the energy policy paradigm of South Korea has focused on developing green energy, several algae biofuel projects have been conducted. As a result, hundreds of domestic microalgae strains have been isolated and some of them were further investigated for biomass or biodiesel potential [10].

Although a number of microalgae strains have already been isolated from various environments around the world, the isolation and characterization of indigenous microalgae species that adapted to local environments are still important for a successful and economically viable microalgae based biodiesel industry. The present study aimed to screen a suitable microalga strain which can be used as a local biodiesel feedstock in South Korea. Based on the biomass and lipid productivity of isolated axenic microalgae strains, Desmodesmus sp. KAERINJ5 was chosen and optimum photoautotrophic growth conditions for biomass and lipid production were investigated.

\section{Materials and Methods}

\section{Isolation, cultivation, and lipid content measurement of the newly isolated microalgae strains}

Freshwater samples were collected from local freshwater reservoirs located in Naejang Mountain National Park, Jeonbuk, Republic of Korea (35 30 '53.8”N, 126 54'11.3'E). Serially diluted freshwater samples were plated on Bold's basal medium (BBM) [11] agar containing meropenem (Sigma-Aldrich, USA) at a concentration of $10 \mathrm{mg} / \mathrm{l}$. Axenic microalgae cultures were obtained by repeated BBM agar plate streaking and microscopic observation.

All media were autoclaved for sterilization at $121^{\circ} \mathrm{C}$ for $20 \mathrm{~min}$. In all routine flask cultivations, the cells were incubated in an orbital shaker with shaking (150 rpm) at $25^{\circ} \mathrm{C}$. Also illumination (the light/dark periods were 12:12) was provided with an equal ratio of warm and cool LED lights at an irradiance of $100 \mu \mathrm{mol} \mathrm{m} \mathrm{s}^{-2}$.

For rapid measurement of microalgae lipid content, a modified Nile red staining method developed by Ren et al. [12] was employed. Axenic microalgal strains were grown in BBM medium with shaking (150 rpm) and illumination (the light/dark periods were $12: 12$ ) at $25^{\circ} \mathrm{C}$ for 7 days. Cells $(5 \mathrm{ml})$ were harvested by centrifugation at 6,000 rpm for $10 \mathrm{~min}$ and washed with sterile distilled water 3 times. Then $5 \mathrm{ml}$ of cell suspensions were pretreated with a sonicator (Fisher Scientific, USA) and mixed with $15 \mu$ l of Nile red (Sigma-Aldrich, USA) solution ( $1 \mathrm{mg} / \mathrm{ml}$ in acetone). The suspensions were stood in the dark for $5 \mathrm{~min}$ and fluorescence intensity was measured using a spectrofluoremeter (Jasco, Japan). The excitation and emission wavelengths were 530 and $568 \mathrm{~nm}$, respectively. The determination of relative fluorescence intensity was obtained by subtracting the intensity from the cell and Nile red alone.

\section{Cloning and sequencing of the ITS sequence}

Genomic DNA was extracted using DNeasy Plant Mini kit (Qiagen, Germany). The ITS region was amplified with the primers ITS1 (5'-TCCGTAGGTGAACCTGCGG-3') and ITS4 (5'-TCCTCCGCTTATTGATATGC-3'). The amplified fragments were purified using the QIAquick PCR purification kit (Qiagen, Germany) and cloned into a pGEM-T Easy vector (Promega, USA) in $E$. 
coli. The nucleotide sequences were determined by the dideoxy method using the SP6 (5'TATTTAGGTGACACTATAG-3') and T7 (5'-TAATACGACTCACTATAGGG3 ') promoter primers of the vector. The ITS sequences of the novel strains were deposited into GenBank under the accession numbers KJ958905, KP406623, KP406624, and KP406626, respectively.

\section{Phylogenetic analysis}

Basic local alignment search tool (BLAST) was used to compare the ITS rDNA sequences of newly isolated novel microalgae strains to sequences in the GenBank database and the most closely related 10 species were selected for phylogenetic analysis. Unclassified sequences were not included. The sequences were manually edited with BioEdit software [13] to trim the non-overlapping 5' and 3 ' regions for the purpose of restricting the multiple sequence alignment within the minimum common region. Those edited sequences aligned using the CLUSTAL W and Neighbor-joining algorithm in MEGA 6 software were used to construct the phylogenetic tree $[14,15]$. Kimura's two-parameter model of sequence evolution was used for calculating the distance matrix [16]. Tree reliability was evaluated by a bootstrap analysis of 1,000 replicates [17].

\section{Microscopic observation}

Light and fluorescence micrographs were acquired using a microscope with a DP73 digital camera (BX53F, Olympus, Japan) at $1,000 \times$ magnification. For lipid droplet observation, cells were stained with $1 \mathrm{mg} / \mathrm{l}$ Nile red (Sigma-Aldrich, USA) dissolved in acetone.

\section{Preliminary study on the growth conditions}

Unialgal isolates were cultivated in $500 \mathrm{ml}$ flasks containing $100 \mathrm{ml}$ of BBM [11], Kuhl [18], BG11 [19] or N8 [20] medium for 7 days to find an appropriate growth medium. In order to figure out the approximate limitation of environmental factors on the biomass productivity of Desmodesmus sp. KAERI-NJ5, the growth of the strain was measured in various conditions. The temperatures used in this study were $15,25,30,35$, and $40^{\circ} \mathrm{C}$; the light intensities were $0,50,100,200$, and $400 \mu \mathrm{mol}$ $\mathrm{m}^{-2} \mathrm{~s}^{-1}$; the $\mathrm{pH}$ values were 5.0, 6.0, 7.0, 8.0, and 9.0; and the $\mathrm{KNO}_{3}$ concentrations were $0.05,0.1,0.5,1.0,5.0$, and $10 \mathrm{~g} / \mathrm{l}$. All cultivations were performed in $500 \mathrm{ml}$ flasks containing $100 \mathrm{ml}$ of medium with agitation (150 rpm).

\section{Measurement of biomass and total lipid content}

The microalgal growth was determined by measuring the optical density of the culture broth using a UV-Vis spectrophotometer at $680 \mathrm{~nm}$ (Biochrome, UK) with the corresponding blank media as the control. Microalgal biomass was calculated by the following regression equation: $\mathrm{y}=0.3605 \mathrm{x}-0.4843\left(\mathrm{R}^{2}=0.9994, p<0.05\right)$, where $\mathrm{y}(\mathrm{g} / \mathrm{l})$ is the cell dry weight (CDW) and $\mathrm{x}$ is the absorbance of the suspension at $680 \mathrm{~nm}$. For the CDW measurement of microalgal biomass, the microalgal culture was filtrated through a GF/C glass fiber filter (GE Healthcare, UK), and the filter containing the biomass was dried in a forced convection oven (JEIO TECH) at $70^{\circ} \mathrm{C}$ to a constant weight.

After cultivation, the algal cells were harvested by centrifugation (4,000 rpm) for $15 \mathrm{~min}$, and the cell pellets were freeze-dried in a high vacuum for 2 days. Then, $0.2 \mathrm{~g}$ of dried microalgae was mixed with $0.5 \mathrm{ml}$ of distilled water and $3 \mathrm{ml}$ of chloroform/methanol (2:1, v/v). The mixture was then shaken for $20 \mathrm{~min}$, centrifuged (10,000 rpm) for $10 \mathrm{~min}$ and the chloroform phase was collected in a pre-weighed tube. The samples were extracted five times and dried in a dry block bath (MG2000, EYELA, Japan) by purging with nitrogen gas at $60^{\circ} \mathrm{C}$. Lipid content was expressed as a percentage (weight of lipid/weight of biomass $\times \%$ ).

\section{Analysis of fatty acids composition}

Fatty acids were extracted from $50 \mathrm{mg}$ of a dried algal cell with $1 \mathrm{ml}$ of $\mathrm{NaOH}-\mathrm{CH}_{3} \mathrm{OH}$. To each aliquot, $0.1 \mathrm{ml}$ of an internal standard of $10 \mathrm{~g} / \mathrm{l}$ tridecanoicacid (C13:0) dissolved in chloroform was added for fatty acid quantification. The mixture was shaken for $10 \mathrm{~min}$ at $75^{\circ} \mathrm{C}$ in a water bath, and cooled to room temperature. Then, $2 \mathrm{ml}$ of boron trifluoride-methanol solution (1:2, v/v) was added, and the mixture was shaken for $10 \mathrm{~min}$ at $75^{\circ} \mathrm{C}$ and cooled to room temperature. Later, $0.3 \mathrm{ml}$ of saturated salt solution was added to make a layered solution. Subsequently, $2 \mathrm{ml}$ of hexane was added, and the mixture was centrifuged. The upper fatty acids layer was subjected to GC-linked mass spectrometry (GC-MS) analysis on an Agilent HP 6890N machine equipped with a 5975 inert mass selective detector. A capillary column DB-5MS (length $30 \mathrm{~m}$, I.D. $0.25 \mathrm{~mm}$, thickness 
$0.25 \mu \mathrm{m}$, Agilent Technologies, USA) was used. The sample size was $1 \mu$, the carrier gas was helium at a flow rate of $1.0 \mathrm{ml} / \mathrm{min}$, the solvent delay time was $5 \mathrm{~min}$, and the injection temperature was $250^{\circ} \mathrm{C}$. The initial oven temperature was $130^{\circ} \mathrm{C}$ for $1 \mathrm{~min}$, and it was increased to $200^{\circ} \mathrm{C}$ at a rate of $5^{\circ} \mathrm{C} / \mathrm{min}$, which was maintained for $5 \mathrm{~min}$. Fatty acids were identified by a direct comparison of their mass spectral pattern and retention index with the NIST 05 mass spectral database. Data are reported as an average of triplicate measurements.

\section{Design of experiments with operational parameters}

Photobioreactors (FMT DS-D, Fermentec, Korea) equipped with custom-made rectangular cool and warm white LED panel modules (OD-TECH, Korea) were used in this study. Kuhl medium (1.5 L) [18] and a 12/12-h on/ off light cycle $\left(200 \mu \mathrm{mol} \mathrm{m}^{-2} \mathrm{~s}^{-1}\right)$ with continuous agitation $(200 \mathrm{rpm})$ and aeration with filtered $(0.22 \mu \mathrm{m})$ ambient air (1 vvm, volume air per volume media per min) were maintained as the basal condition. Inoculum was prepared in the photobioreactor containing $1.5 \mathrm{~L}$ of the basal medium at the basal condition for 7 days. The inoculum concentration was about $5 \times 10^{7}$ cells per liter medium.

A face-centered central composite design with three variables was used to determine the effect of the variables on the production of the algal biomass. The Design Expert software version 9 (Stat-Ease Inc., USA) was used for random experimental design, statistical analysis and prediction of response. The chosen variables were temperature, $\mathrm{pH}$ and concentration of nitrogen source $\left(\mathrm{KNO}_{3}\right)$ in the medium. The levels of $\mathrm{pH}$, temperature and nitrogen source concentration were determined based on the results from "Effect of culture conditions on phototrophic growth of Desmodesmus sp. KAERI-NJ5" in the 'Result and Discussion' section. For this model, 20 experiments were carried out including six replicates at the central point to evaluate the pure error and the predicted value of the optimum biomass production and culture conditions obtained. All experiments were carried out in duplicate and the average of the biomass productivity was taken as the response. The standard deviation was less than $3 \%$. According to the experimental results, the relationship between the variables and the response was calculated by the seconddegree polynomial equation as below:

$$
\begin{aligned}
Y & =\beta_{0}+\beta_{1} \mathrm{~A}+\beta_{2} \mathrm{~B}+\beta_{3} \mathrm{C}+\beta_{1} \beta_{2} \mathrm{AB}+\beta_{1} \beta_{3} \mathrm{AC} \\
& +\beta_{2} \beta_{3} \mathrm{BC}+\beta_{1} \beta_{1} \mathrm{~A}^{2}+\beta_{2} \beta_{2} \mathrm{~B}^{2}+\beta_{3} \beta_{3} \mathrm{C}^{2}
\end{aligned}
$$

where $Y$ is the response variable; $\beta_{0}$ is the intercept (constant); $\beta_{1}, \beta_{2}$ and $\beta_{3}$ are linear coefficients; $\beta_{1} \beta_{2}$, $\beta \beta_{1} \beta_{3}$, and $\beta_{2} \beta_{3}$ are interaction coefficients; $\beta_{1} \beta_{1}, \beta_{2} \beta_{2}$, and $\beta_{3} \beta_{3}$ are squared coefficients; and $A, B, C, A B, A C$, $B C, A^{2}, B^{2}$ and $C^{2}$ are the levels of independent variables. The quality of the fitting was expressed by the coefficient of determining $\mathrm{R}^{2}$ and $\mathrm{R}_{\text {adjust, }}^{2}$ and its statistical significance was determined by Fisher's $F$-test value. Model terms were selected or rejected based on the $P$ value with a $99 \%$ confidence level. The fitted polynomial equation was then expressed in the form of contour and surface plots in order to visualize individual and interactive effect variables on the responses within the design. The experimental value from the optimum condition was compared with the predicted value of the biomass production to verify the model of the response surface.

\section{Effect of nitrogen starvation on lipid content and compo- sitions}

A microalgal cell was grown at $29^{\circ} \mathrm{C}$ with a modified Khul medium ( $\mathrm{pH}$ 6.5, $0.5 \mathrm{~g} / \mathrm{l}$ of $\mathrm{KNO}_{3}$ ) for 7 days in a photobioreactor. The culture was equally divided into two samples and harvested by centrifugation $(12,000$ rpm) for $15 \mathrm{~min}$. One of the samples was resuspended with the same volume of the modified Khul medium, while the other was resuspended with the same volume of the nitrogen-free modified Khul medium. The resuspended cells were re-incubated for 3 days and harvested as described above. The lipid content and compositions were analyzed as described above.

\section{Results and Discussion}

\section{Isolation and identification of novel microalgae}

In order to isolate novel microalgae candidates for biomass and lipid production, 115 axenic green microalgae strains were isolated from the local freshwater reservoirs after a few cycles of agar plate spreading and microscopic observation. The relative fluorescence intensity of the axenic strains was attained by the ultrasonicassisted method [12]. Among them, 17 photoautotrophic green microalgae strains were selected based on their fluorescence intensity ( $\leq 500$ a.u.). The ITS sequences of 
Table 1. ITS sequencing result and closest relatives of the novel microalgae strains.

\begin{tabular}{|c|c|c|c|c|}
\hline Strains & Genbank accession number & Number of ITS sequences & Closest relatives & Identity (\%) \\
\hline \multirow[t]{3}{*}{ Dictyosphaerium sp. KAERI-NJ2 } & KP406626 & 754 & Dictyosphaerium sp. CCAP 222/43 & 92 \\
\hline & & & Closteriopsis acicularis & 84 \\
\hline & & & Marasphaerium gattermannii & 82 \\
\hline \multirow[t]{3}{*}{ Desmodesmus sp. KAERI-NJ5 } & KJ958905 & 602 & Desmodesmus sp. GM4a & 94 \\
\hline & & & Desmodesmus asymmetricus & 94 \\
\hline & & & Desmodesmus communis & 90 \\
\hline \multirow[t]{3}{*}{ Dunaliella sp. KAERI-NJ23 } & KP406624 & 599 & Dunaliella sp. FL1 & 90 \\
\hline & & & Dunaliella salina & 89 \\
\hline & & & Dunaliella bardawil & 89 \\
\hline \multirow[t]{3}{*}{ Chlorella sp. KAERI-NJ24 } & KP406623 & 712 & Chlorella sp. CCCryo297-06 & 92 \\
\hline & & & Didymogenes anomala & 88 \\
\hline & & & Didymogenes palatina & 88 \\
\hline
\end{tabular}

the selected strains were determined to locate their taxonomic positions (Table 1). Based on the ITS sequence identity $(\leq 95 \%)$ and molecular phylogenetic analysis (Fig. 1), four strains were identified as novel strains and tentatively named as in Table 1. Among them, Dunaliella sp. KAERI-NJ23 showed the lowest sequence identity when compared with the known microalgae species in the GenBank database until now. However, all the strains shared the highest ITS sequence identity with uncharacterized strains. Thus, more specific phylogenetic characterization cannot be given.

\section{Phototrophic growth characteristics and lipid contents of the novel strains}

Four widely used microalgae growth media including BBM [11], Kuhl [18], BG11 [19] and N8 [20] medium were chosen to figure out suitable a medium for their phototrophic growth (Fig. 2A). Chlorella sp. KAERINJ24 and Dunaliella sp. KAERI-NJ23 showed the highest microalgal optical density when cultivated with the BG11 and BBM medium, respectively. Also, the Khul medium was the most suitable medium for Desmodesmus sp. KAERI-NJ5 and Dictyosphaerium sp. KAERI-NJ2 cultivation. Although a slight difference in the algal biomass was observed, Desmodesmus sp. KAERI-NJ5 was found to be the most suitable strain for algal biomass production. The TL and FAMEs contents of the algal biomass cultivated with the medium that showed the highest growth was determined (Fig. 2B). Desmodesmus sp. KAERI-NJ5 and Chlorella sp. KAERI-NJ24 showed the highest TL and FAMEs contents, respectively. In general, the production of biodiesel from algae is dependent on the microalgal biomass production rate and lipid content. When considering the growth of the microalgae strains, Desmodesmus sp. KAERI-NJ5 was found to be the most ideal strain for the lipid production of biodiesel. Recently, species of the genus Desmodesmus were studied intensively in tropical Taiwan, and regarded as promising microalgae for biodiesel production [24, 2629]. Therefore, Desmodesmus sp. KAERI-NJ5 was deposited as KCTC 18331P in the Korean Collection for Type Cultures and used for further study.

\section{Effect of culture conditions on the phototrophic growth of Desmodesmus sp. KAERI-NJ5}

Numerous environmental factors influence the growth of microalgae [21]. Also, many reports have revealed that the growth rate and lipid content of microalgae have a close relationship with these factors, especially light, nitrogen, phosphorus, $\mathrm{pH}$, and temperature $[3,6$, $21,22]$. Before optimizing the biomass and lipid production, the effects of light intensity, nitrogen concentration, $\mathrm{pH}$, and temperature on the strain were evaluated (Fig. 3).

The growth measurement of the strain KAERI-NJ5 showed that the optimum growth temperature was between 30 and $35^{\circ} \mathrm{C}$ (Fig. $3 \mathrm{~A}$ ). However, the growth rate was significantly reduced at $15^{\circ} \mathrm{C}$ and the cell concentration grown at $40^{\circ} \mathrm{C}$ gradually decreased after 5 days of incubation. In general, the optimum growth tempera- 


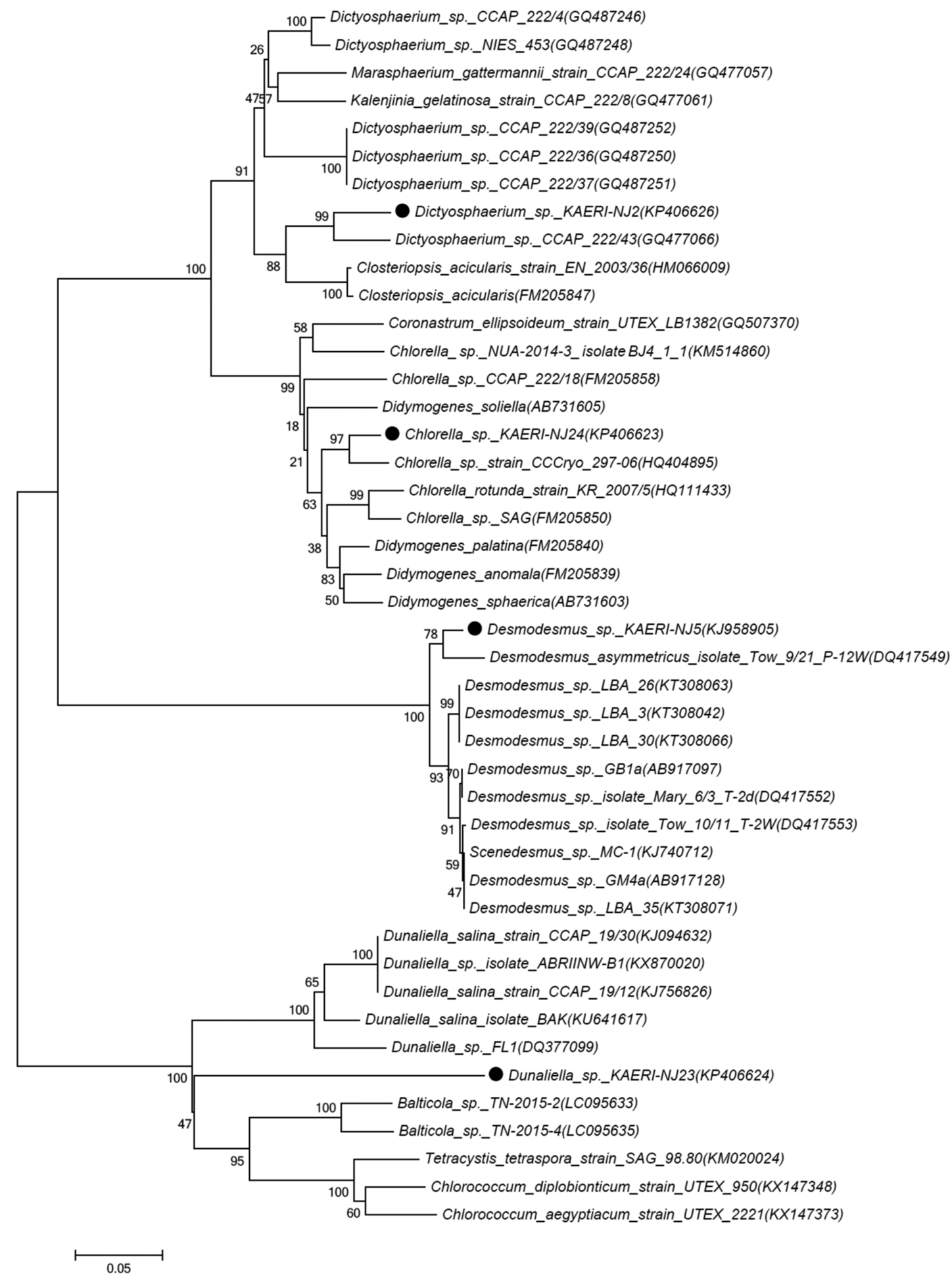

Fig. 1. Evolutionary relationships of the novel strains. The evolutionary history was inferred using the Neighbor-Joining method [21]. The optimal tree with the sum of branch length $=1.94331905$ is shown. The percentage of replicate trees in which the associated taxa clustered together in the bootstrap test (1000 replicates) is shown next to the branches [24]. The tree is drawn to scale, with branch lengths in the same units as those of the evolutionary distances used to infer the phylogenetic tree. The evolutionary distances were computed using the Kimura 2-parameter method [23] and are in the units of the number of base substitutions per site. Evolutionary analyses were conducted in MEGA6 [22]. 

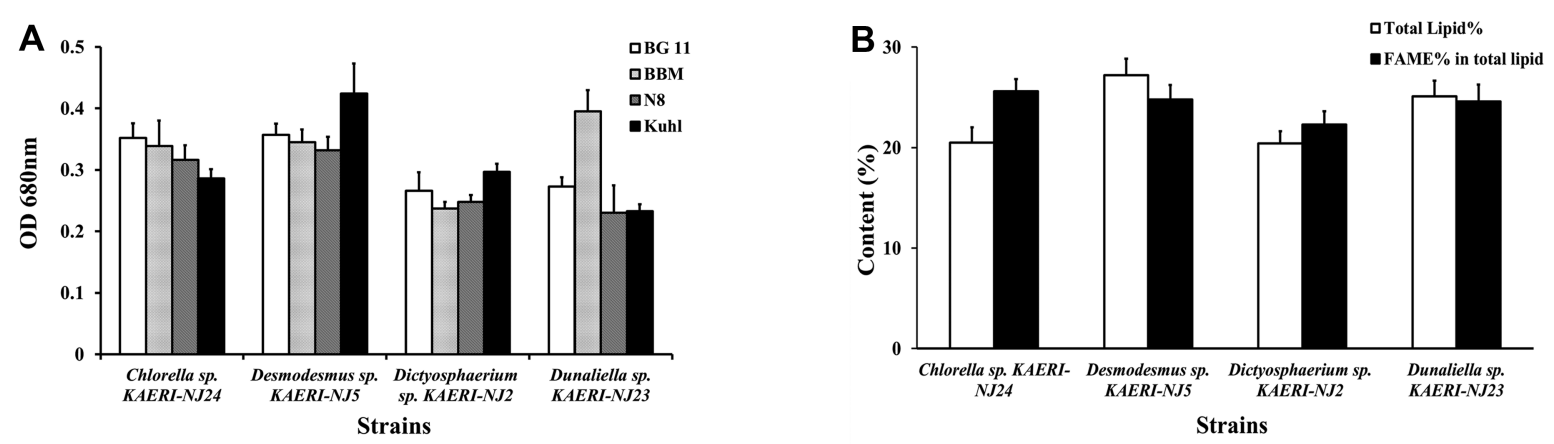

Fig. 2. Total lipid and FAMEs contents of the microalgae strains. (A) Measurement of the microalgal biomass after cultivation for 7 days in 4 different growth media. (B) TL and FAMEs contents of the microalgae strains after cultivation for 7 days in the most suitable medium for each strain. Data are represented as the average of triplicate measurements.

ture was between 20 and $25^{\circ} \mathrm{C}$ for most algae [23]. However, some Desmodesmus species isolated in subtropical and tropical zones exhibited thermo-tolerance [24]. Although the cell concentration grown at $40^{\circ} \mathrm{C}$ slightly decreased after 5 days of incubation, the strain KAERINJ5 could be classified as a thermo-tolerant microalgae when considering the isolated location and temperature preference for its growth. As shown in Fig. 3B, the growth of the strain KAERI-NJ5 showed clear dependency on the light intensity. In our culture condition, more illumination resulted in a higher biomass concentration. Although the maximum growth was observed at
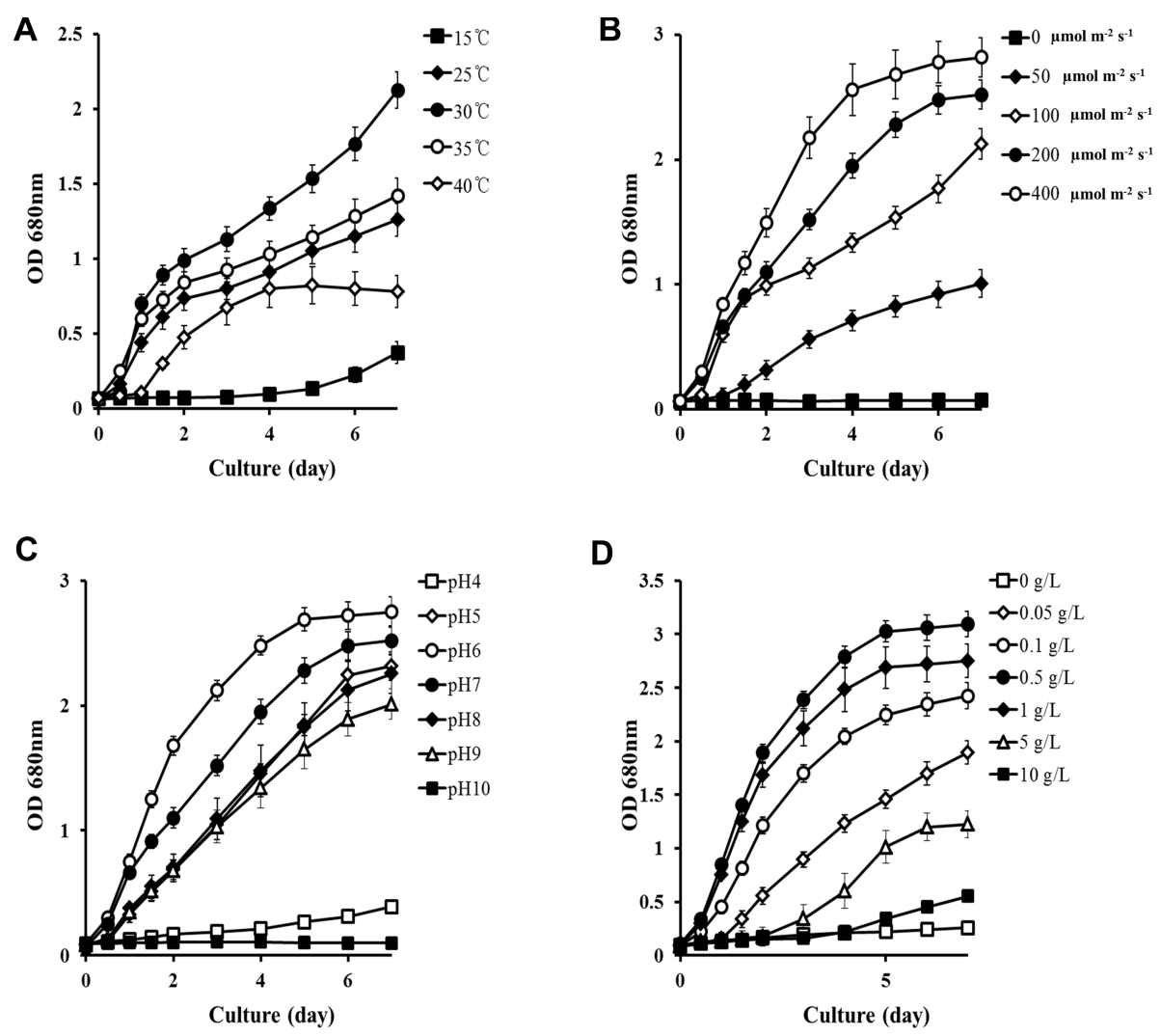

Fig. 3. Effect of various environmental factors on the growth of Desmodesmus sp. KAERI-NJ5. (A) temperature, (B) light intensity, (C) $\mathrm{pH},(\mathrm{D})$ concentration of $\mathrm{KNO}_{3}$. Data are represented as the average of triplicate measurements. 
$400 \mu \mathrm{mol} \mathrm{m}{ }^{-2} \mathrm{~s}^{-1}$, the time-course growth pattern and biomass productivity were similar to that of culture grown with $200 \mu \mathrm{mol} \mathrm{m} \mathrm{m}^{-2}$. The strain showed a similar growth pattern in a wide range of $\mathrm{pH}$ ranges from $\mathrm{pH} 5$ and $\mathrm{pH} 9$ (Fig. 3C). Although the strain could barely grow at $\mathrm{pH} 4$, the strain could not grow over $\mathrm{pH} 10$, and there was no living cell after 7 days of incubation. The amount of nitrogen required for the growth of the strain was investigated, and the culture with the medium containing $0.5 \mathrm{~g} / \mathrm{l}$ of $\mathrm{KNO}_{3}$ showed the highest growth (Fig. 3D). Increasing the concentration of $\mathrm{KNO}_{3}$ from 0 to $1 \mathrm{~g} / \mathrm{l}$ promoted the growth of the strain, but a $\mathrm{KNO}_{3}$ concentration more than $5 \mathrm{~g} / \mathrm{l}$ caused growth retardation. Based on the result above, we concluded that the strain requires specific ranges of the environmental growth factors in our experimental conditions except light intensity. Therefore, we excluded the effect of the light intensity for later experiments, and light intensity was maintained at $200 \mu \mathrm{mol} \mathrm{m}{ }^{-2} \mathrm{~s}^{-1}$.

According to several reports, the Desmodesmus spe- cies were described as environmental stress tolerant microalgae [24, 26-29]. Based on the above results, Desmodesmus sp. KAERI-NJ5 was also flexible in different culture conditions, and which supported previous studies. However, a more specific culture condition is needed to increase biomass productivity.

\section{Modeling of biomass production by Desmodesmus sp. KAERI-NJ5}

The RSM approach is a sequential and exploratory method that establishes the relationship between more than one variable and a given response. This method is highly useful and is often used for optimizing or minimizing various biological processes. In order to maximize the biomass production by the strain KAERI-NJ5, a detailed optimization of chosen phototrophic culture variables, including temperature, $\mathrm{pH}$, and $\mathrm{KNO}_{3}$ concentration, was investigated through an RSM approach, a face centered central composite design. Table 2 presents the 20 experimental runs and predicted resultant values

Table 2. Face centered central composite design with 3 independent variables for biomass production by Desmodesmus sp. KAERI-NJ5.

\begin{tabular}{|c|c|c|c|c|c|c|c|c|c|}
\hline \multirow{2}{*}{ Std } & \multirow{2}{*}{ Run } & \multicolumn{6}{|c|}{ Actual and coded levels of variables } & \multicolumn{2}{|c|}{ CDW $(g / l)$} \\
\hline & & $\mathrm{A}: \mathrm{pH}$ & Code & B: Temperature $\left({ }^{\circ} \mathrm{C}\right)$ & Code & $\mathrm{C}: \mathrm{KNO}_{3}(\mathrm{~g} / \mathrm{l})$ & Code & Results $^{\mathrm{a}}$ & Predicted \\
\hline 13 & 1 & 6 & 0 & 30 & 0 & 0.5 & -1 & 1.45 & 1.4 \\
\hline 1 & 2 & 5 & -1 & 25 & -1 & 0.5 & -1 & 0.1 & 0.12 \\
\hline 20 & 3 & 6 & 0 & 30 & 0 & 1 & 0 & 1.18 & 1.23 \\
\hline 19 & 4 & 6 & 0 & 30 & 0 & 1 & 0 & 1.21 & 1.23 \\
\hline 18 & 5 & 6 & 0 & 30 & 0 & 1 & 0 & 1.22 & 1.23 \\
\hline 5 & 6 & 5 & -1 & 25 & -1 & 1.5 & 1 & 0.16 & 0.15 \\
\hline 6 & 7 & 7 & 1 & 25 & -1 & 1.5 & 1 & 1.29 & 1.28 \\
\hline 3 & 8 & 5 & -1 & 35 & 1 & 0.5 & -1 & 0.2 & 0.21 \\
\hline 7 & 9 & 5 & -1 & 35 & 1 & 1.5 & 1 & 0.25 & 0.24 \\
\hline 12 & 10 & 6 & 0 & 35 & 1 & 1 & 0 & 0.89 & 0.9 \\
\hline 17 & 11 & 6 & 0 & 30 & 0 & 1 & 0 & 1.3 & 1.23 \\
\hline 16 & 12 & 6 & 0 & 30 & 0 & 1 & 0 & 1.19 & 1.23 \\
\hline 2 & 13 & 7 & 1 & 25 & -1 & 0.5 & -1 & 1.32 & 1.33 \\
\hline 14 & 14 & 6 & 0 & 30 & 0 & 1.5 & 1 & 1.34 & 1.39 \\
\hline 11 & 15 & 6 & 0 & 25 & -1 & 1 & 0 & 1.1 & 1.09 \\
\hline 4 & 16 & 7 & 1 & 35 & 1 & 0.5 & -1 & 0.85 & 0.86 \\
\hline 10 & 17 & 7 & 1 & 30 & 0 & 1 & 0 & 1.13 & 1.14 \\
\hline 9 & 18 & 5 & -1 & 30 & 0 & 1 & 0 & 0.24 & 0.24 \\
\hline 15 & 19 & 6 & 0 & 30 & 0 & 1 & 0 & 1.3 & 1.23 \\
\hline 8 & 20 & 7 & 1 & 35 & 1 & 1.5 & 1 & 0.83 & 0.81 \\
\hline
\end{tabular}

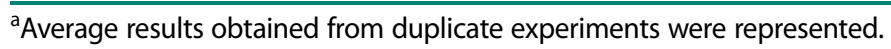


of response. Experiment $13\left(\mathrm{pH} 6,30^{\circ} \mathrm{C}\right.$, and $0.5 \mathrm{~g} / \mathrm{l}$ of $\mathrm{KNO}_{3}$ ) offered the highest biomass production (1.45 g/l), while experiment $1\left(\mathrm{pH} 6,30^{\circ} \mathrm{C}\right.$, and $0.5 \mathrm{~g} / \mathrm{l}$ of $\left.\mathrm{KNO}_{3}\right)$ showed the lowest biomass production $(0.1 \mathrm{~g} / \mathrm{l})$. Based on the experimental results and multiple regression analysis from the data shown in Table 2, a mathematical regression model fitted in terms of coded factors was established as follows:

$$
\begin{aligned}
& \text { Biomass production }(\mathrm{g} / \mathrm{l})=1.231+0.447 \mathrm{~A}-0.095 \mathrm{~B} \\
& -0.005 \mathrm{C}-0.14 \mathrm{AB}-0.02 \mathrm{AC}+2.267 \mathrm{E}-016 \mathrm{BC} \\
& -0.542 \mathrm{~A}^{2}-0.232 \mathrm{~B}^{2}+0.168 \mathrm{C}^{2}
\end{aligned}
$$

A positive or a negative sign before a term indicates a synergistic and antagonistic effect, respectively. The equation of the model indicates that biomass production has both linear and quadratic relations with the variables used in this study.

\section{Statistical analysis of the model}

Parameters, including $F$-value, $p$-value, lack of fit, and coefficients were evaluated by analysis of variance (ANOVA) for the model of biomass production (Table 3).
The model $F$-value of 217.08 implies that most of the variations in the response can be explained by the model. The associated Prob $>F$ ( $p$-value) is used to determine whether the $F$-value is high enough to verify statistical significance or not and a $p$-value $<0.01$ indicates that the model is regarded as significant at a $>99 \%$ confidence level. A low lack of fit $F$-value (0.4661) indicates the lack of fit was insignificant relative to the pure error. In addition, when evaluating the associated $p$ value (0.789), there was a $78.9 \%$ chance that this lack of fit value occurred due to noise from biomass production.

The adequacy of the model was also checked by calculating the correlation coefficient of determination $\left(\mathrm{R}^{2}=0.9949\right)$, indicating that $99.49 \%$ of the variability in the response could be explained by this model. The high value of the adjusted determination coefficient $\left(\mathrm{R}^{2}{ }_{\text {adj }}=\right.$ 0.9903) and reasonable agreement with the predicted coefficient $\left(\mathrm{R}_{\text {pred }}^{2}=0.9829\right)$ implied that the model fit the experimental data well. Hence, all the coefficients of determination indicated that the model is highly reliable upon repetition of the experiments. The coefficient of

\begin{tabular}{|c|c|c|c|c|c|c|}
\hline Source & Sum of squares & $\mathrm{DOF}^{\mathrm{a}}$ & Mean square & F-value & $p$-value (Prob $>\mathrm{F}$ ) & Significance ${ }^{b}$ \\
\hline Model & 4.1056 & 9 & 0.4562 & 217.0751 & $<0.0001$ & $\sqrt{ }$ \\
\hline$A: p H$ & 1.9981 & 1 & 1.9981 & 950.8129 & $<0.0001$ & $\sqrt{ }$ \\
\hline B: Temperature $\left({ }^{\circ} \mathrm{C}\right)$ & 0.0903 & 1 & 0.0903 & 42.9464 & $<0.0001$ & $\sqrt{ }$ \\
\hline $\mathrm{C}: \mathrm{KNO}_{3}(\mathrm{~g} / \mathrm{l})$ & 0.0002 & 1 & 0.0002 & 0.1190 & 0.7373 & - \\
\hline$A B$ & 0.1568 & 1 & 0.1568 & 74.6150 & $<0.0001$ & $\sqrt{ }$ \\
\hline$A C$ & 0.0032 & 1 & 0.0032 & 1.5228 & 0.2454 & - \\
\hline BC & 0.0000 & 1 & 0.0000 & 0.0000 & 1 & - \\
\hline$A^{2}$ & 0.8087 & 1 & 0.8087 & 384.8117 & $<0.0001$ & $\sqrt{ }$ \\
\hline$B^{2}$ & 0.1484 & 1 & 0.1484 & 70.6007 & $<0.0001$ & $\sqrt{ }$ \\
\hline$C^{2}$ & 0.0774 & 1 & 0.0774 & 36.8146 & $<0.0001$ & $\sqrt{ }$ \\
\hline Residual (total error) & 0.0210 & 10 & 0.0021 & - & - & - \\
\hline Lack of fit & 0.0067 & 5 & 0.0013 & 0.4661 & 0.789 & $\begin{array}{c}- \\
\text { (not significance) }\end{array}$ \\
\hline Pure error & 0.0143 & 5 & 0.0029 & - & - & - \\
\hline$R^{2}$ & \multicolumn{2}{|c|}{0.9949} & $\mathrm{R}_{\mathrm{adj}}^{2}$ & \multicolumn{2}{|c|}{0.9903} & \\
\hline $\mathrm{R}_{\text {pred }}^{2}$ & \multicolumn{2}{|c|}{0.9829} & $\mathrm{CV} \%{ }^{\mathrm{e}}$ & \multicolumn{2}{|c|}{4.94} & \\
\hline
\end{tabular}
variation $(\mathrm{CV})$ is the ratio of pure error of estimate to the

Table 3. ANOVA of the fitted quadratic polynomial model for biomass production.

${ }^{\mathrm{a}}$ Degree of freedom.

${ }^{b}$ At $99 \%$ confidence.

'Adjusted determination coefficient.

${ }^{\mathrm{d}}$ Predicted determination coefficient.

${ }^{e}$ Coefficient of variation. 
mean value of the resulting response, indicating reproducibility of the model. In general, a model with no greater than $10 \%$ of $\mathrm{CV}$ can be regarded as a reproducible model. Therefore, the CV value $(\mathrm{CV} \%=4.94)$ of the model indicates a highly reliable reproducibility of the experiments.

In this model, the linear terms for $\mathrm{pH}$ (A) and temperature $(B)$, the square terms for all variables $\left(\mathrm{A}^{2}, \mathrm{~B}^{2}\right.$, $\mathrm{C}^{2}$ ), and the interactive terms for $\mathrm{pH}$ and temperature (AB) were found to be significant at the $1 \%$ probability level (Table 3). When considering the $F$ - and $p$-value, temperature is the most important factor for its biomass production. In addition, synergistic interaction between temperature and $\mathrm{pH}$ could be explained by the high $F$ and low $p$-value. Taken all together, this model is significant and desirable to navigate the design space.

\section{Analysis by response surface methodology}

The interactions between the independent variables on the biomass production were graphically observed by response surface plots and contour plots (Fig. 4). These plots were created by taking two variables at a time while the remainder of the variables were kept at the ' 0 ' level.

Fig. 4A shows the effects of growth temperature and $\mathrm{pH}$ individually and their interaction on biomass pro- duction at a fixed nitrogen concentration. The biomass production initially increased and then slowly reduced by further increasing these parameters. The results indicated that a high temperature and $\mathrm{pH}$ were not advantageous for biomass production. The obvious elliptical contour plot indicated a significant interaction between these two growth parameters. Also, the highest response value $(\mathrm{CDW}=1.4 \mathrm{~g} / \mathrm{l})$ was achieved when the $\mathrm{pH}$ was around 6.5 and the temperature was around $29^{\circ} \mathrm{C}$. Although the similar individual effects of $\mathrm{pH}$ and temperature on the biomass production could be seen in Fig. $4 \mathrm{~B}$ and $4 \mathrm{C}$, the nitrogen concentration did not show any correlation with other parameters. In addition, the contour plots showed a clear diagonal pattern, indicating an absence of mutual interaction. When considering the ANOVA and RSM results of the model, there was no linear effect from nitrogen concentrations used in the experiment.

When considering the number of published studies, the genus Desmodesmus is not a quite well-known microalga for biomass and lipid production. Only several reports are available, and these studies regarded Desmodesmus sp. as thermo tolerant microalgae [24, 2629]. However, the optimum temperature for the biomass production of the strain was between 25 and $35^{\circ} \mathrm{C}$. In addition, other environmental factors, including light
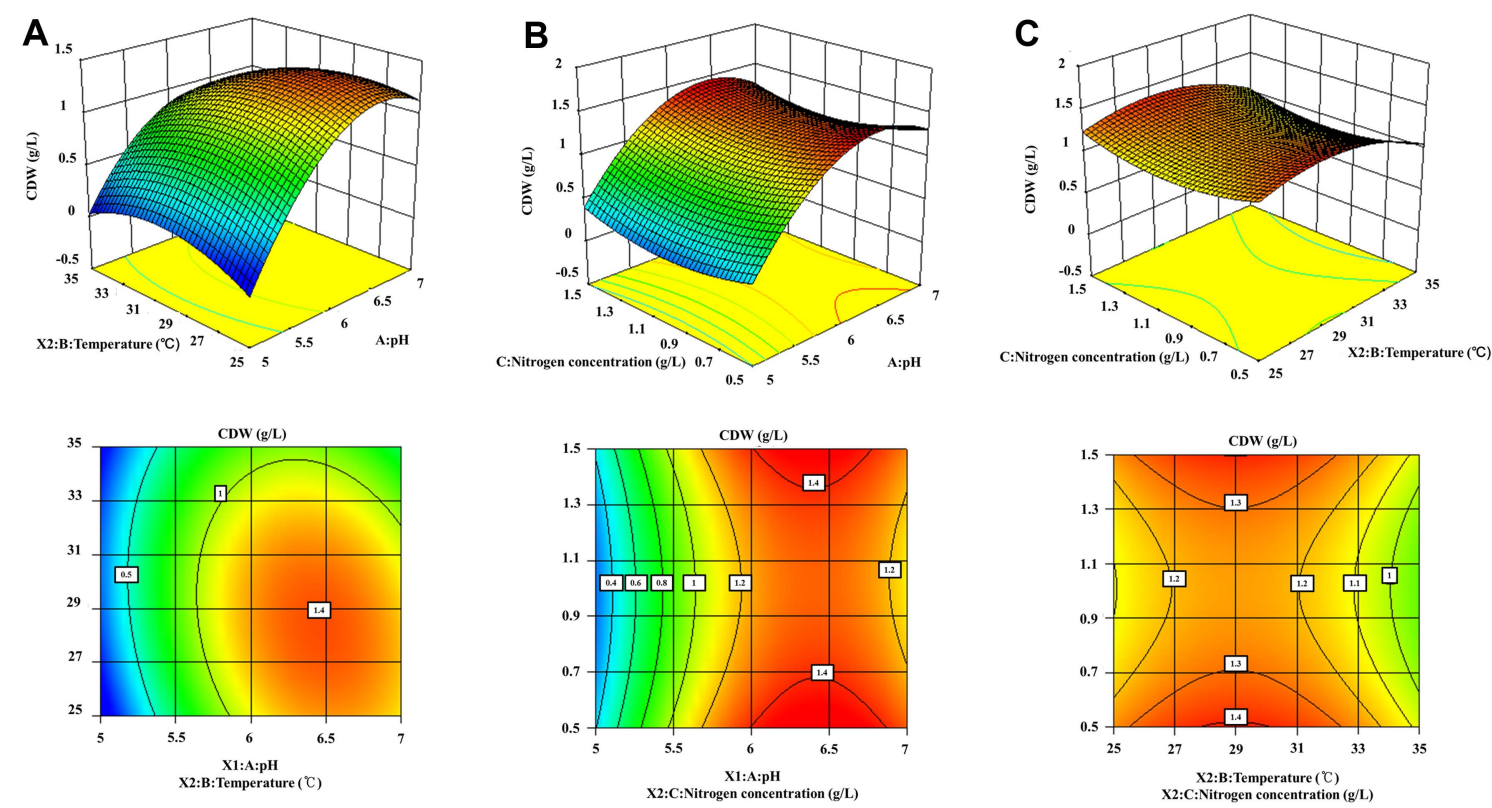

Fig. 4. Three-dimensional response surface plots (up) and the contours plots (down) for dry cell weight showing the interactions effect. (A) temperature and $\mathrm{pH}(\mathrm{B})$ nitrogen concentration and $\mathrm{pH}(\mathrm{C})$ nitrogen concentration and temperature. 
Table 4. Optimum conditions selected by desirability function and validation of the experimental model.

\begin{tabular}{|c|c|c|c|c|c|c|}
\hline & $\mathrm{pH}$ & Temperature $\left({ }^{\circ} \mathrm{C}\right)$ & $\begin{array}{l}\text { Nitrogen concentration } \\
\qquad(\mathrm{g} / \mathrm{l})\end{array}$ & $\begin{array}{l}\text { Predicted CDW } \\
(\mathrm{g} / \mathrm{l})\end{array}$ & Desirability & $\begin{array}{l}\text { Experimental CDW } \\
(\mathrm{g} / \mathrm{l})^{\mathrm{a}}\end{array}$ \\
\hline 1 & 6.46 & 29.18 & 0.51 & 1.52 & 1 & $1.55 \pm 0.24$ \\
\hline 2 & 6.54 & 27.66 & 0.52 & 1.51 & 1 & $1.52 \pm 0.37$ \\
\hline 3 & 6.52 & 27.59 & 0.52 & 1.51 & 1 & - \\
\hline 4 & 6.38 & 29.09 & 0.53 & 1.50 & 1 & - \\
\hline 5 & 6.37 & 29.71 & 0.51 & 1.50 & 1 & - \\
\hline
\end{tabular}

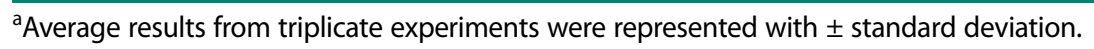

intensity, night and day period, $\mathrm{pH}$, nitrogen sources, and nitrogen concentration, were also important for their growth and lipid production. And Desmodesmus sp. KAERI-NJ5 also showed light intensity-dependent increase of biomass production (Fig. 3B). However, the degree of importance of these factors was different species-to-species to some extent. In the case of Desmodesmus sp. KAERI-NJ5, the RSM study revealed that the $\mathrm{pH}$ of the medium was the main factor for biomass production (Table 3 and Fig. 4A).

\section{Experimental validation of the model}

Based on the RSM results, five optimum conditions for biomass production were suggested by the "Numerical Optimization" tool of the Design Expert software (Table 4). Among them, two conditions were chosen to verify the statistical model. Validation under optimum conditions was carried out in triplicate. The experimental biomass yields from the two conditions showed close agreement with the predicted yields, indicating the high accuracy and reliability of the fitted model (Table 4).

\section{Effect of nitrogen starvation on lipid production and com- position}

Generally, nitrogen starvation is a crucial factor for increasing the lipid content of microalgal biodiesel feedstock [3]. The basic principle is that an insufficient nitrogen source for protein synthesis is required for cell growth, which leads to excess carbon produced by photosynthesis to storage molecules, such as lipid or starch. In addition, lipids and starch can be easily packed in cells and can also be used to survive in stressed environments [30]. However, the effect of nitrogen starvation on the FAMEs composition is species dependent [31]. Therefore, variation on the lipid production and FAMEs com- position by nitrogen limitation of Desmodesmus sp. KAERI-NJ5 should be analyzed in detail.

The size of lipid droplet was significantly increased by nitrogen starvation, indicating an accumulation of lipid molecules (data not shown). The TL and FAMEs content highly increased 1.86- and 2.31-fold by nitrogen starva-

Table 5. Effect of nitrogen starvation on lipid content and FAMEs profile of Desmodesmus sp. KAERI-NJ5.

\begin{tabular}{|c|c|c|}
\hline \multirow[b]{2}{*}{ FAMEs } & \multicolumn{2}{|c|}{ Relative FAMEs content (\%) } \\
\hline & Control & $\begin{array}{l}\text { Nitrogen } \\
\text { starvation }\end{array}$ \\
\hline Palmitic acid (C16:0) & $29.70 \pm 1.59$ & $46.17 \pm 2.75$ \\
\hline Palmitoleic acid (C16:1) & $8.60 \pm 0.49$ & $1.30 \pm 0.13$ \\
\hline Hexadecadienoic acid (C16:2) & $4.35 \pm 0.24$ & $0.54 \pm 0.04$ \\
\hline Hexadecatrienoic acid (C16:3) & $3.20 \pm 0.12$ & $0.31 \pm 0.01$ \\
\hline Stearic acid (C18:0) & $2.94 \pm 0.11$ & $2.12 \pm 0.11$ \\
\hline Oleic acid (C18:1) & $29.40 \pm 1.12$ & $39.43 \pm 1.99$ \\
\hline Linoleic acid (C18:2) & $6.87 \pm 0.65$ & $3.02 \pm 0.15$ \\
\hline Linolenic acid (C18:3) & $0.00 \pm 0.01$ & $3.02 \pm 0.21$ \\
\hline others & $14.94 \pm 0.92$ & $4.10 \pm 0.31$ \\
\hline Saturated fatty acids content ${ }^{b}$ & $47.70 \pm 3.12$ & $48.82 \pm 2.37$ \\
\hline Monounsaturated fatty acids ${ }^{c}$ & $37.60 \pm 2.09$ & $40.73 \pm 2.11$ \\
\hline Polyunsaturated fatty acids ${ }^{d}$ & $14.42 \pm 1.07$ & $6.89 \pm 0.59$ \\
\hline FAME content $\left(\% \text { of } C D W^{e}\right)^{f}$ & $21.20 \pm 1.45$ & $49.00 \pm 2.49$ \\
\hline Total lipid content $(\% \text { of } C D W)^{g}$ & $28.00 \pm 1.19$ & $52.02 \pm 3.88$ \\
\hline
\end{tabular}

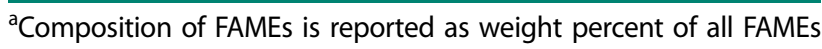
content.

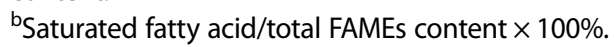

${ }^{c}$ Monounsaturated fatty acid/total FAMEs content $\times 100 \%$.

${ }^{\mathrm{d}}$ Monounsaturated fatty acid/total FAMEs content $\times 100 \%$.

${ }^{e}$ Cells were harvested at stationary phase before loss of cell mass, lyophillized and used for lipid analysis.

fTotal fatty acids/CDW $\times 100 \%$.

${ }^{9}$ Total lipids/CDW $\times 100 \%$.

All data are represented as the average of triplicate measurements with standard deviation. 
tion, respectively (Table 5). Their weight also accounted for 49 and $52.02 \%$ of the CDW. The FAMEs analysis revealed that the main fatty acid components of the strain were fatty acids with C16-C18. Nitrogen starvation highly increased the amount of palmitic, oleic, and linolenic acid. Also, the major storage lipid molecules were palmitic and oleic acid, which have a similar lipid profile to palm oil, indicating a potential viable biodiesel feedstock. At the same time, the content of other kinds of lipid molecules were reduced, thereby indicating the preference of lipid storage. Microalgal fatty acid composition is an indicator of suitability as biodiesel feedstock and economic viability. In general, a higher amount of PUFAs in a biodiesel indicates a higher tendency of the biodiesel to oxidize. According to the FAMEs profile of the strain, only $6.89 \%$ of the PUFAs remained after nitrogen starvation. In addition, oleic acid, which is known to balance the oxidative stability and low temperature fluidity of biodiesel, was the main unsaturated fatty acid, as it accounted for over $39 \%$ of the total fatty acids content. Furthermore, saturated fatty acids comprised $48.82 \%$ of the total fatty acids content. These saturated fatty acids increase oxidative stability and cetane number, an indicator of biodiesel quality, thereby indicating high potential for biodiesel feedstock [32]. When considering the FAMEs composition of the strain, the biomass from the strain can be used as a biodiesel feedstock with good quality, and nitrogen starvation was useful for lipid production and fatty acid quality. Although biomass, lipid and FAMEs productivities of Desmodesmus sp. KAERI-NJ5 were not the highest among the species in the genus Desmodesmus [24, 2629] and its close genus Scenedesmus [12, 32], the results were comparable with strains belongs to these genera and could be increased by further optimization of other environ factors, including light intensity, $\mathrm{CO}_{2}$ purging and carbon source supplementation.

\section{Acknowledgment}

This work was supported by the research grant from the Korea Atomic Energy Research Institute, Jeongeup, Republic of Korea.

\section{Conflict of Interest}

The authors have no financial conflicts of interest to declare.

\section{References}

1. Lang X, Dalai AK, Bakhshi NN, Reaney MJ, Hertz PB. 2001. Preparation and characterization of bio-diesels from various bio-oils. Bioresour. Technol. 80: 53-62.

2. Greenwell HC, Laurens LML, Shields RJ, Lovitt RW, Flynn KJ. 2010. Placing microalgae on the biofuels priority list: a review of the technological challenges. J. R. Soc. Interface 7: 703-726.

3. Scott SA, Davey MP, Dennis JS, Horst I, Howe CJ, Lea-Smith DJ, et al. 2010. Biodiesel from algae: challenges and prospects. Curr. Opin. Biotechnol. 21: 277-286.

4. Chisti Y. 2008. Biodiesel from microalgae beats bioethanol. Trends Biotechnol. 26: 126-131.

5. Li YG, Xu L, Huang YM, Wang F, Guo C, Liu CZ. 2011. Microalgal biodiesel in China: Opportunities and challenges. Appl. Energy 88: $3432-3437$.

6. Chaichalerm S, Pokethitiyook P, Yuan W, Meetam M, Sritong K, Pugkaew W, et al. 2012. Culture of microalgal strains isolated from natural habitats in Thailand in various enriched media. Appl. Energy 89: 296-302.

7. Sharma YC, Singh V. 2017. Microalgal biodiesel: A possible solution for India's energy security. Renew. Sustain. Energy Rev. 67: 7278.

8. Sharma YC, Singh B, Upadhyay SN. 2008. Advancements in development and characterization of biodiesel: A review. Fuel 12: 2355-2373.

9. Chung WS, Kim SS, Moon KH, Lim CY, Yun SW. 2017. A conceptual framework for energy security evaluation of power sources in South Korea. Energy 137: 1066-1074.

10. Hong JW, Jo SW, Yoon HS. 2015. Research and development for algae-based technologies in Korea: a review of algae biofuel production. Photosynth. Res. 123: 297-303.

11. Nichols HW, Bold HC. 2007. Trichosarcina polymorpha Gen. et Sp. Nov. J. Phycol. 1: 34-38.

12. Ren HY, Liu BF, Ma C, Zhao L, Ren NQ. 2013. A new lipid-rich microalga Scenedesmus sp. strain R-16 isolated using Nile red staining: effects of carbon and nitrogen sources and initial $\mathrm{pH}$ on the biomass and lipid production. Biotechnol. Biofuels 6: 143.

13. Hall TA. 1999. BioEdit: a user-friendly biological sequence alignment editor and analysis program for Windows 95/98/NT. Nucleic Acids Symp. Ser. 41: 95-98.

14. Saitou N, Nei M. 1987. The neighbor-joining method: a new method for reconstructing phylogenetic trees. Mol. Biol. Evol. 4: 406-425.

15. Tamura K, Stecher G, Peterson D, Filipski A, Kumar S. 2013. MEGA6: Molecular Evolutionary Genetics Analysis Version 6.0. Mol. Biol. Evol. 30: 2725-2729.

16. Kimura M. 1980. A simple method for estimating evolutionary rates of base substitutions through comparative studies of nucleotide sequences. J. Mol. Evol. 16: 111-120.

17. Felsenstein J. 1985. Confidence limits on phylogenies: An approach using the bootstrap. Evolution 39: 783-791. 
18. Prescott DM. 1964. Methods in cell physiology. pp. 159-187. In Kuhl A, Lorenzen $\mathrm{H}$ (eds), Handling and culturing of Chlorella, 1st Ed. Academic Press, New York and London.

19. Rippka R, Herdman H. 1992. Pasteur Culture Collection of Cyanobacteria. pp. 103. Catalogue \& Taxonomic Handbook. Institut Pasteur, Paris.

20. Vonshak A, Richmond A. 1986. Handbook of microalgal mass culture. pp. 117-145. CRC Press, Boca Raton Florida.

21. Zhu J, Rong J, Zong B. 2013. Factors in mass cultivation of microalgae for biodiesel. Chinese J. Catal. 34: 80-100.

22. Khozin-Goldberg I, Cohen Z. 2006. The effect of phosphate starvation on the lipid and fatty acid composition of the fresh water eustigmatophyte Monodus subterraneus. Phytochemistry 67: 696701.

23. Butterwick C, Heaney SI, Talling JF. 2005. Diversity in the influence of temperature on the growth rates of freshwater algae, and its ecological relevance. Freshw. Biol. 50: 291-300.

24. Pan YY, Wang ST, Chuang LT, Chang YW, Chen CNN. 2011. Isolation of thermo-tolerant and high lipid content green microalgae: Oil accumulation is predominantly controlled by photosystem efficiency during stress treatments in Desmodesmus. Bioresour. Technol. 102: 10510-10517.

25. Chiu PH, Soong K, Chen CNN. 2016. Cultivation of two thermotolerant microalgae under tropical conditions: Influences of carbon sources and light duration on biomass and lutein productivity in four seasons. Bioresour. Technol. 212: 190-198.
26. Rios LF, Martinez A, Klein BC, Wolf Maciel MR, Maciel Filho R. 2018. Comparison of growth and lipid accumulation at three different growth regimes with Desmodesmus sp. Waste and Biomass Valorization 9: 421-427.

27. Ji F, Hao R, Liu Y, Li G, Zhou Y, Dong R. 2013. Isolation of a novel microalgae strain Desmodesmus sp. and optimization of environmental factors for its biomass production. Bioresour. Technol. 148: 249-254.

28. Ho SH, Chang JS, Lai YY, Chen CNN. 2014. Achieving high lipid productivity of a thermotolerant microalga Desmodesmus sp. F2 by optimizing environmental factors and nutrient conditions. Bioresour. Technol. 156: 108-116.

29. Rios LF, Klein BC, Luz LF, Maciel Filho R, Wolf Maciel MR. 2015. Nitrogen Starvation for Lipid Accumulation in the Microalga Species Desmodesmus sp. Appl. Biochem. Biotechnol. 175: 469-476.

30. Courchesne NMD, Parisien A, Wang B, Lan CQ. 2009. Enhancement of lipid production using biochemical, genetic and transcription factor engineering approaches. J. Biotechnol. 141: 3141.

31. Hempel N, Petrick I, Behrendt F. 2012. Biomass productivity and productivity of fatty acids and amino acids of microalgae strains as key characteristics of suitability for biodiesel production. J. Appl. Phycol. 24: 1407-1418.

32. Wu H, Miao X. 2014. Biodiesel quality and biochemical changes of microalgae Chlorella pyrenoidosa and Scenedesmus obliquus in response to nitrate levels. Bioresour. Technol. 170: 421-427. 